\title{
La estrategia de retorno de la industria española: El caso del sector calzado en Alicante, su importancia y determinantes ${ }^{*}$
}

\author{
CARMEN MARTÍNEZ MORA a , FERNANDO MERINO DE LUCAS ${ }^{\text {b }}$ \\ a Universidad de Alicante, Facultad de Económicas y Empresariales, Aptdo. 99, 03080 Alicante, \\ España.E-mail: cmmora@ua.es \\ b Universidad de Murcia, Facultad de Economía y Empresa, Campus del Espinardo, 30100 Murcia, \\ España.E-mail: fmerino@um.es
}

\section{RESUMEN}

Este trabajo analiza los procesos de retorno al país de origen de algunas de las fases del proceso productivo que previamente se habían deslocalizado a países extranjeros en el sector del calzado. La metodología empleada consiste en un estudio de casos a empresas del sector. Los resultados del estudio revelan que la estrategia de relocalización responde a los cambios en el entorno de las empresas derivados de la reciente crisis económica y que no estaban presentes cuando se adoptaron las decisiones de deslocalización. En el nuevo contexto, la reducción de los plazos de entrega y los aumentos de flexibilidad en la distribución se revelan como las principales razones que motivan el aumento de producción nacional y la disminución en el extranjero.

Palabras clave: Relocalización, Deslocalización, competitividad, calzado.

\section{Reshoring the Spanish Production of Footwear: Its Importance and Determinants}

\begin{abstract}
This paper analyzes the processes of return to the country of origin of some of the phases of the productive process that in the past had been relocated to foreign countries in the footwear sector. The methodology used is a case study with qualitative information from companies in the sector The results of the study reveal that the relocation strategy adopted responds to changes in the business environment resulting from the recent economic crisis that were not present when the delocalization strategy was adopted. In the new economic situation, the need of more reduced delivery times as well as flexibility in the distribution are revealed as the reasons that justify the increase in domestic production and the reduction of production abroad.
\end{abstract}

Keywords: Reshoring, Offshoring, Competitiveness, Footwear.

Clasificación JEL: F23, L67, F13

\footnotetext{
* Los autores agradecen la colaboración de los directivos de las empresas estudiadas al proporcionarles la información precisa. Cualquier error derivado del análisis o estudio de estos datos es de la exclusiva responsabilidad de los autores.
}

Artículo recibido en marzo de 2017 y aceptado en mayo de 2017

Artículo disponible en versión electrónica en la página www.revista-eea.net, ref. ə-35302 


\section{INTRODUCCIÓN}

Uno de los cambios más importantes que ha tenido lugar en la estructura productiva mundial durante los últimos años del siglo XX y los primeros del siglo XXI, ha sido el proceso de deslocalización de actividades productivas desde países avanzados hacia países con un menor nivel de desarrollo. Este desplazamiento ha estado motivado, en muchos casos, por las ventajas de costes que estas economías presentaban. Ello generó una preocupación importante por la pérdida de puestos de trabajo en los países de origen dada la dificultad de competir con los menores costes laborales de las economías a las que se dirigían, aun teniendo en cuenta los diferentes niveles de productividad (Wright, 2014, Gagliardi, Iammarino y Rodríguez-Pose, 2015, etc.) así como por su impacto sobre otras actividades de la empresa como es el caso de la innovación (Mitza y Kreuzer, 2015) o el propio crecimiento económico (Alan, 2004). Por su parte, para las economías de acogida este proceso supone entradas adicionales de capital y tecnología que les permite avanzar en su senda de desarrollo y mejorar sus expectativas de crecimiento (Sanchez-Robles, 2006, o Hansen, SchaumburgMüller y Pottenger, 2008).

Sin embargo, recientemente se viene detectando la existencia de casos de empresas que deshacen esta senda, es decir que habiendo desplazado su producción a países con menores costes laborales en un pasado reciente, deciden recuperarla en los países de origen de dichas empresas ${ }^{1}$. Este fenómeno, al que cabe llamar relocalización, ha despertado un cierto interés en los responsables políticos de algunos países ${ }^{2}$ en la medida en que contribuye a recuperar actividades manufactureras en las economías avanzadas, con claros efectos positivos en términos de creación de empleo y por sus interrelaciones con el resto de la economía (Christopherson et al., 2014). Los casos de relocalización son relativamente escasos y repartidos entre sectores y países diversos, lo que ha dificultado su estudio desde un marco de análisis integrado que establezca sus factores determinantes. Además, la literatura existente sobre este tema ha señalado bastantes elementos que todavía necesitan un desarrollo más profundo (véase, desde una perspectiva teórica Fratocchi et al., 2014, y Stentoft et al., 2016 sobre los estudios empíricos). En cualquier caso, la literatura que ayuda a entender la deslocalización puede proporcionar también los fundamentos para abordar el estudio de esta deslocalización en sentido contrario.

En este trabajo se presenta un estudio de los fenómenos de relocalización de

\footnotetext{
${ }^{1}$ Véase el informe de Boston Consulting Group que concluye que más de un tercio de las grandes empresas manufactureras están considerando llevar de nuevo la producción a EEUU que desplazaron a China.

2 A modo de ejemplo el "Insourcing American Jobs Forum" del presidente Obama en EEUU.
} 
un sector como el calzado que en el pasado reciente fue en gran medida deslocalizado hacia China. La fabricación de calzado es intensiva en mano de obra, los acuerdos internacionales redujeron las barreras internacionales a la importación de calzado proveniente de China en las economías avanzadas (a partir de su ingreso en la WTO en 2001) y las reducciones en los costes de transporte supusieron un factor estimulante de ese proceso de deslocalización de la producción. Como es bien sabido, España ha tenido tradicionalmente una industria productora de calzado relativamente importante y competitiva en los mercados internacionales con empresas mayoritariamente locales. Dichas empresas no fueron ajenas a las ventajas competitivas que suponía la producción en Asia y a mediados de los años noventa y principios del 2000 deslocalizaron gran parte de su producción hacia aquellas economías. Sin embargo, como ha ocurrido en otros países y sectores en los últimos años se observan casos de relocalización de estas actividades hacia España, por lo que parece constituir un ejemplo interesante para abordar el fenómeno de la relocalización.

Dadas las características del fenómeno (casos más o menos aislados sin una literatura previa que haya podido proporcionar un marco de análisis integrado) y la heterogeneidad de las empresas del sector, la metodología utilizada es el estudio de casos para poner de relieve cuáles pueden ser los factores que han podido determinar la adopción de estrategias de relocalización por parte de las empresas del sector. Eisenhardt (1989) señala que el estudio de casos es una metodología adecuada para el desarrollo posterior de marcos teóricos especialmente cuando se trata de temas o situaciones nuevas. Por otro lado, esta aproximación permite detectar la relevancia de factores explicativos de dicho proceso, lo que puede ser de interés para futuros estudios de otros sectores y que pudieran quedar omitidos con un enfoque agregado. Hay que tener en cuenta que la mayoría de las estadísticas existentes (datos de flujos de inversión directa o de importaciones) no permiten capturar este efecto. Asimismo esta metodología de casos ha sido utilizada en algunos de los trabajos previos para otros países, unas veces a través del análisis de un solo caso y otras con varios, como en el presente estudio (véase Gylling et al., 2015, Moser, 2013).

Los resultados que se obtienen en este trabajo pueden contribuir a la elaboración de un marco de análisis que contemple el conjunto de factores que explican el fenómeno de la relocalización. En los diversos trabajos previos se obtienen algunas causas determinantes, en unos casos coincidentes con las que aquí se presentan y en otros diferentes. Los motivos de la relocalización no son los mismos en todas las industrias ni para todas las empresas, aunque algunos elementos coincidan, por lo que el análisis de una gran diversidad de casos se hace necesario para poder descifrarlos todos de forma general.

La estructura del trabajo es la siguiente: La sección segunda revisa la literatura económica y la evidencia empírica previa relativa a los fenómenos de 
deslocalización y outsourcing internacional y, en particular, la que contempla los procesos de replanteamiento de la estrategia. La sección tercera describe cuáles han sido las empresas analizadas en este trabajo y su justificación, así como las estrategias generalizadas de deslocalización y relocalización adoptadas en el sector. Seguidamente, se detalla la metodología empleada para abordar el análisis. En la sección quinta se presentan las principales características de los casos analizados, para recoger en la sección sexta las conclusiones del estudio y las cuestiones pendientes a abordar en futuras investigaciones.

\section{MARCO TEÓRICO Y EVIDENCIA EMPÍRICA PREVIA}

Desde la perspectiva neoclásica, las actividades productivas se localizan en aquellos emplazamientos que tengan ventajas competitivas para su producción. Así, las actividades en las que los recursos naturales tienen un papel importante en su desarrollo, tendrán determinada su localización por factores que podrían considerarse ajenos a la dinámica de los mercados, aunque un análisis más profundo revelaría que detrás de esta consideración lo que se esconde en muchos casos son unos costes de transporte elevados. Más allá de estas actividades, las diferencias en los costes de producción (en gran medida determinados por la dotación de factores de producción) y en la proximidad a los mercados se han considerado tradicionalmente como los elementos determinantes de los patrones de localización de las actividades productivas.

Sin embargo, el coste de los factores de producción y los costes de transporte no son suficientes para explicar la localización de la producción en las economías más avanzadas, sino que otros elementos se han desvelado igualmente importantes. En primer lugar, la existencia de externalidades en la localización y aglomeración supone una ventaja adicional para la elección de un determinado lugar para llevar a cabo el proceso productivo. Este elemento (inicialmente estudiado por Marshall, Arrow y Romer) ha sido desarrollado ampliamente por la literatura, tanto teórica como empírica, inspirando el diseño de políticas que promovieran la concentración geográfica al reforzarse el atractivo de un emplazamiento por la existencia de una masa crítica de empresas del mismo sector y/o sectores vinculados a éste (Delgado, Porter y Stern, 2010).

La literatura económica ha ido utilizando técnicas diversas para determinar la importancia de distintos elementos para afectar al atractivo de cada lugar como emplazamiento para la actividad productiva. A modo de resumen, puede señalarse que factores como la existencia de economías de aglomeración, la existencia de mano de obra cualificada, y los costes de distintos factores productivos (trabajo, energía, etc.) quedan claramente confirmados como factores relevantes de la localización (véase Arauzo-Carod, Liviano-Solís y Manjón-Antolín, 2010). Dado que estos factores no son constantes, sino que pueden variar (p.ej. el diferencial en los costes de los inputs se verá afectado por la evolución del mismo en los dos 
países así como por el tipo de cambio entre ellos), el atractivo relativo de dos ubicaciones se verá alterado y el retorno al lugar original siempre resultará más fácil en la medida en que los costes de transacción serán más reducidos que los de una tercera alternativa.

Partiendo de este punto, Baldwin y Venables (2013) desarrollan un modelo interesante donde incluyen explícitamente el coste de la disgregación geográfica de las distintas fases del proceso productivo. Su modelo tiene en cuenta que hay distintos tipos de procesos (el tipo "araña" donde los distintos componentes se producen por separado con una fase final de ensamblaje y el modelo "serpiente" donde la producción tiene lugar de forma gradual y aditiva sobre el output de la fase previa, siendo posibles las combinaciones de ambos). A partir de este marco, muestran cómo los cambios en estos costes ligados a la disgregación modifican la fragmentación geográfica del proceso productivo pudiéndose generar discontinuidades motivadas por el desplazamiento de las fases ligadas al ensamblaje que arrastran a otras fases al existir una ventaja derivada de estar geográficamente próximas. En este modelo, la relocalización (desplazamiento de fases del proceso productivo que previamente se llevaron a otro país) puede explicarse tanto por los cambios en el diferencial de costes entre los dos países como por los costes derivados de dicha disgregación geográfica, pudiendo generarse cambios no monotónicos por las razones expuestas.

El fenómeno de la relocalización que se estudia en ese trabajo puede encontrar su acomodo teórico dentro del existente para explicar la localización de las actividades productivas o el cambio de ésta (véase, por ejemplo, Ellram, Tate y Petersen, 2013). Más allá de los casos de relocalización que pudieran explicarse como la corrección de errores de decisiones previas de deslocalización, serán los cambios en los costes de transporte (en un sentido amplio), en el diferencial de costes de producción los potencialmente explicativos desde un punto de vista teórico. Por otra parte, la existencia de externalidades (positivas) derivadas de la existencia de otras empresas y que puedan actuar como un freno a la hora de deslocalizarse, pueden serlo también a la hora de relocalizarse si en el emplazamiento de origen despareció totalmente el tejido productivo que genera dichas externalidades. Por el contrario, si en el emplazamiento original permanece alguna de esas fuentes de externalidad (como por ejemplo la existencia de otras empresas que no hubieran deslocalizado), el factor externalidades supondrá un menor freno para el proceso de retorno que para el de ida.

En muchos casos, la deslocalización está vinculada a procesos de modificación de los límites de las empresas, esto es, la externalización de partes del proceso productivo. De hecho, el marco teórico para la inversión directa internacional que supone el paradigma OLI (Dunning, 1980), propone que la internacionalización a través de inversión en el extranjero tiene lugar cuando existen, además de ventajas de propiedad y localización, ventajas de 
internalización, es decir, cuando los costes de transacción asociados a la externalización de actividades en otros países son superiores a los de internalizarlas mediante procesos de inversión directa. Por tanto, a la hora de explicar el proceso de deslocalización-relocalización hay que incluirlo en un marco que contemple la externalización de la actividad productiva. Williamson (1975) plantea la Teoría de los Costes de Transacción donde justifica la presencia de un conjunto de tareas dentro de la empresa (frente a su alternativa que es la contratación con terceros) en la existencia de costes de transacción. En estos costes se incluyen no sólo la búsqueda de los proveedores, sino también el seguimiento de los contratos, la vigilancia para evitar filtraciones de información específica de las empresas, la realización de controles de calidad, los costes adicionales de trasmitir elementos relevantes para el proceso productivo, etc. De hecho, distintos trabajos empíricos para la economía española (véase Myro et al., 2008, o Myro y Labrador, 2008) señalan que en muchos casos los procesos de internacionalización de empresas españolas llevan asociado un proceso de externalización de las actividades productivas. En este aspecto hay que señalar que los costes de transacción serán probablemente no superiores en el caso de la relocalización que en la deslocalización debido a la mayor cercanía psíquica y cultural con agentes del propio país que con otros de un país lejano.

La relocalización supone, por tanto, una reconsideración de las decisiones de outsourcing internacional tomadas en los años anteriores. Como ha sido analizado en otros trabajos (Freytag et al., 2012), esta reconsideración no tiene porqué explicarse necesariamente por problemas derivados del desarrollo de la estrategia, aunque, por ejemplo Kinkel (2012) detecta que la mayoría de los casos que se han producido en Alemania responden a esta situación. Veltri et al. (2008) señalan como factor explicativo la aparición de cambios externos ante los cuales la vuelta a fabricar en el país nacional pasa a ser una estrategia competitiva. En todo caso, hay que destacar que este cambio no implica que se sustituya totalmente la subcontratación de toda la producción en el extranjero. Si bien hay algunas empresas que sí que lo hacen, la situación generalizada consiste en una combinación del mantenimiento de la práctica de outsourcing internacional con relocalizaciones parciales de producción en España o países cercanos geográficamente.

Debe tenerse presente que la literatura citada hace referencia a los factores determinantes de procesos de localización y deslocalización, sin embargo la relocalización es un fenómeno distinto, en el sentido de que siempre es posterior a decisiones tomadas en el pasado por las empresas. En este sentido y en relación con la evidencia empírica existente, hay que señalar que a pesar de la ausencia de un marco teórico específico y a lo reciente de la estrategia, en la última década la investigación de la misma ha sido muy importante pudiéndose identificar en torno 
a una veintena de trabajos publicados desde 2009 hasta $2016^{3}$, correspondiendo la mayor parte de ellos a los años posteriores a 2014.

Como se ha señalado, las estadísticas de comercio internacional o de inversión directa no permiten valorar la extensión de los fenómenos de relocalización, puesto que estos quedan incluidos en un conjunto mucho más amplio de flujos de comercio e inversión. Sin embargo, a partir del análisis de los datos de cadenas de valor globales y las Tablas Input-Output mundiales, Timmer et al. (2016) consiguen tener una aproximación de las importaciones consecuencias de los procesos de fragmentación internacional del proceso productivo. Sus resultados muestran que, si bien entre 2000 y 2008 la fragmentación internacional crecía, entre este año y 2014 se ha producido un retroceso apreciable.

Para conocer con más detalle las causas y las estrategias de relocalización se hace necesaria una perspectiva centrada en la empresa. Existen diferentes trabajos publicados sobre la relocalización que analizan las causas y la entidad de la estrategia para diferentes industrias y países. Fratocchi et al. (2016), a partir de una amplia y exhaustiva recopilación de casos de fuentes secundarias, muestra que sectores como el del textil, la electrónica y la mecánica son los que concentran un mayor número de casos, y que la zona en la que, con mayor frecuencia, se produce la deslocalización es China, mientras que en los países del este de Europa o África se producen muy pocos casos. Con respeto a la zona de retorno, Europa es más frecuente que Norteamérica. En esta línea, un trabajo interesante es el de Stentoft et al. (2016) que realiza una amplia revisión de la literatura existente, detectando veinte trabajos sobre el tema. En los mismos, se analizan casos en países tales como Dinamarca, Nueva Zelanda, Estados Unidos, Corea del Sur, Reino Unido o España y en diversos sectores de manufacturas, tales como automóviles o bicicletas. La metodología empleada en los trabajos citados comprende estudios conceptuales, en los que se define y analiza el significado de la estrategia de relocalización, estudios de casos, de encuestas y mixtos, con información cualitativa y cuantitativa, en los que se analizan básicamente las razones de la estrategia. Finalmente otros estudios analizan también las cuestiones relacionadas con la decisión de las empresas de llevar a cabo procesos de relocalización (véase Ancarini et al., 2015) a partir de muestras de empresas que realizan procesos de desinversión. Las conclusiones del conjunto de los trabajos publicados señalan que los principales motivos de la relocalización están relacionados con la búsqueda de mejoras en costes, calidad, plazos y flexibilidad, riesgos y conservación y ampliación de los mercados.

\footnotetext{
${ }^{3}$ En Stentoft. et al. (2016) se encuentra una completa revisión de la evidencia empírica existente.
} 


\section{EMPRESAS DEL SECTOR DEL CALZADO ANALIZADAS Y ESTRATEGIAS DE DESLOCALIZACIÓN Y RELOCALIZACIÓN}

La producción de calzado en España se encuentra localizada en muy pocas regiones (Comunidad Valenciana, Castilla-La Mancha, La Rioja, Baleares, Aragón y Murcia). Entre éstas, destaca la Comunidad Valenciana donde se concentran dos terceras partes de la producción total española, y en particular, la provincia de Alicante, donde se concentra el 64 por ciento de la producción nacional, así como las empresas con mayor liderazgo del sector.

Desde sus orígenes, las empresas del sector de esta zona geográfica se han distinguido por su importante orientación exportadora basada en una competitividad derivada fundamentalmente de los precios. Dicha ventaja competitiva de las empresas españolas del sector se debía, fundamentalmente, a unos costes laborales inferiores a los de otros países, lo cual puede constituir una ventaja competitiva en un producto como el calzado que es intensivo en factor trabajo.

Al igual que en otros países europeos, la competitividad vía precio fue debilitándose progresivamente, hasta que a mediados de los años noventa la permanencia del sector en el mercado nacional e internacional, se encontraba seriamente amenazada. La amenaza venía, en gran medida por la aparición de países emergentes con menores costes de producción a lo que se añadía las estrategias de deslocalización en estos países que estaban adoptando los principales competidores europeos. En resumen, la globalización del sector, en gran medida impulsada por las ventajas de precios (aunque no exclusivamente, Lowder 1999) reconfiguraba el mapa de la producción de calzado dejando a España y a otros países europeos en una situación de escasa competitividad en el mercado internacional.

Ante ello, la estrategia generalizada de las empresas líderes alicantinas, desde mediados de los años noventa hasta principios del 2000, consistió en la deslocalización de una parte o toda su producción en los mismos países que los principales competidores internacionales, fundamentalmente en el sudeste asiático. Dicha deslocalización se combinaba con el mantenimiento de parte de la producción en España (fundamentalmente la del producto de mayor calidad y prestigio) y el lanzamiento de nuevas líneas de gama media y baja producidas en países con menores costes laborales. El nuevo panorama supuso que aquellas empresas que fabricaban calzado de gama media deslocalizaron todo su proceso productivo mientras que aquellas cuya única línea de producto era de alta gama, y no introdujeron otras nuevas, mantuvieron la fabricación en España. Esta situación es semejante a la que se ha producido en este sector productivo en Italia (Amighini y Rabellotti, 2010) aunque en este país el proceso de deslocalización tuvo una fase importante en la que algunos países del Este de 
Europa acogieron distintas fases de la producción antes de acabar en países del sudeste asiático (Cutrini, 2011).

La práctica totalidad de la estrategia de deslocalización se llevó a cabo a través de la subcontratación de la fabricación del producto en dichos países sin realizar inversiones directas en el exterior significativas. Es decir, a través de prácticas de outsourcing internacional. El objetivo era ganar competitividad en precio, reduciendo costes de fabricación para poder mantener las ventas, y concentrar las inversiones en lo que pasaría a constituir su "núcleo de negocio": distribución, marketing, diseño y calidad (de acuerdo con los enfoques teóricos de Barney, 1991, Arnold, 2000, y Gadde y Jonsson, 2009). Como consecuencia de esta estrategia gran parte de la producción de calzado desapareció de España, mientras que las empresas consiguieron mantener sus ventas y posicionamiento en los mercados gracias a la ventaja competitiva que suponía producir en países con menores costes (un estudio detallado de este proceso se encuentra en Fuster, Martínez, y Pardo, 2008) ${ }^{4}$. Asimismo, otro de los efectos de las deslocalizaciones fueron los incrementos de las importaciones de calzado procedentes de estos países para ser exportadas desde España, tal y como sucede en otros sectores industriales de la economía española (Gandoy, 2017).

Sin embargo, la profunda crisis económica de 2007-2013, con la consiguiente reducción de la demanda en el mercado nacional y europeo, que es el principal destino de las exportaciones del sector, unida a otros factores en los países fabricantes, ha puesto en duda la adecuación de seguir produciendo en dichos países. El seguimiento del sector pone de manifiesto que algunas de las principales empresas están haciendo frente a estas dificultades a través de un proceso reciente de relocalización de parte o toda la fabricación en España, el país de origen de la empresa. Dicho fenómeno se detecta, especialmente, a partir de 2010 y 2011.

\section{METODOLOGÍA}

Con el objetivo de identificar toda la casuística que determina las distintas decisiones de relocalización que está adoptando el sector del calzado de la zona seleccionada, la provincia de Alicante, se ha realizado un análisis cualitativo con una metodología de estudio de casos.

Para estudiar fenómenos como el que se aborda en este trabajo (con casos aislados y en el que pueden resultar relevantes un amplio conjunto de factores), la metodología del estudio de casos parece adecuada ya que pondrá de relieve los factores relevantes en la adopción de esta estrategia en cada empresa a la vez

\footnotetext{
${ }^{4}$ Se puede detectar cierta similitud con el proceso que han seguido algunas empresas textiles alemanas en sus procesos de externalización internacional hacia Grecia y FYROM (véase Labrianidis y Kalantaridis, 2004).
} 
que permite enmarcar dichas decisiones en el entorno específico de cada una (Eisendhart, 1989).

Se ha partido de una relación de las empresas más representativas, en términos de volumen de negocio, en el sector del calzado en Alicante que proporciona el Instituto de la Mediana y Pequeña Industria Valenciana (IMPIVA), seleccionándose entre éstas a aquellas que llevaron a cabo procesos de deslocalización en el pasado. La relación de las principales empresas contiene dieciocho casos, de las cuales once deslocalizaron parte de su producción. De estas once, siete están relocalizando parcial o totalmente la fabricación de nuevo en España y cuatro mantienen la producción en el extranjero sin modificaciones relevantes. Se han realizado entrevistas personalizadas a los directores generales o a los directores comerciales de las once empresas seleccionadas, durante los meses de febrero a mayo de 2014. La descripción de dichas entrevistas fue contrastada por los propios entrevistados, incorporándose sus aportaciones y observaciones. Además la información obtenida por esta vía fue complementada con la de los propios informes financieros de las empresas depositados en el Registro Mercantil, así como por publicaciones en prensa, revistas especializadas del sector y noticias publicadas en internet.

El conjunto de las características y las razones que explican la relocalización en las siete empresas que los realizan, se puede describir a través de la presentación de cuatro casos-tipo de empresas que sirven como modelo de todas las situaciones que se están desarrollando. La selección de estas cuatro responde al referido rasgo de cubrir tipos de empresa diferentes a la vez que compartir el patrón de relocalización con otras. Por este motivo se describen por separado cada uno de estos casos, a través de los cuales queda descrita gran parte de la casuística de las decisiones de relocalización del sector.

El contenido de las entrevistas se ha basado en cuestiones relacionadas con las características de las empresas y de sus productos, así como sobre los aspectos relacionados con la distribución, la innovación y la estructura del mercado en el que operan. También se les ha preguntado detalladamente por la entidad, la importancia, las características y las razones de sus procesos de internacionalización a través de sus distintas formas, tales como las exportaciones, acuerdos contractuales y deslocalizaciones. Finalmente las cuestiones planteadas se refieren a las decisiones de relocalización y en particular a sus causas.

\section{CUATRO CASOS DE EMPRESAS DE CALZADO Y SUS DECISIONES DE RELOCALIZACIÓN}

Para enmarcar los casos cuyo análisis se va a presentar, hay que señalar que todas las empresas analizadas subcontrataron parte o toda su fabricación en 
India y China, siendo este último país el que concentra la mayor parte de la producción. Estas empresas, en consonancia con lo que hicieron otras empresas del sector, deslocalizaron a Asia la producción de las líneas de calzado de gama media, incrementando sus inversiones en España en el desarrollo de departamentos de diseño, calidad, marketing y distribución. Este proceso fue unido al mantenimiento de la fabricación en España de las líneas de alta gama, con mayor precio y diferenciación de producto. Puede señalarse que el proceso de outsourcing en el extranjero presentó inicialmente algunos problemas, sobre todo de calidad y plazos de entrega, que se fueron resolviendo progresivamente con el desplazamiento de personal propio de las empresas, técnico y directivos, al país fabricante.

Otro de los rasgos relevantes es que estas empresas se encuentran en el decil superior de tamaño (en términos de ventas) del sector en la provincia de Alicante y combinadas suponen el 7,7\% de las ventas, según la información proporcionada por SABI. Además, casi todas las empresas distribuyen toda la producción desde España y el mercado de exportación supone entre el 50 y el 70 por cien de sus ventas totales, siendo los principales destinos de las ventas el mercado nacional y europeo.

De forma general, puede concluirse que la estrategia de deslocalización permitió alcanzar en casi todos los casos los resultados perseguidos de expansión, gracias a las ganancias de competitividad logradas al aprovechar los menores costes laborales para las líneas de producto cuya fabricación pasó a realizarse en países asiáticos, unido a las mejoras alcanzadas en la calidad, diseño, marketing y distribución, derivadas del incremento de inversión de las empresas en estos departamentos. Sin embargo, en los últimos años se han presentado cambios en el entorno de las empresas, que lo hacen diferente al momento en el que se adoptó la decisión de deslocalizar y en muchos casos no fáciles de prever por parte de las empresas protagonistas. Estos cambios pueden resumirse en:

- Encarecimiento de los costes laborales en China (según la Organización International del Trabajo, los salarios en el sector manufacturero chino se duplicaron entre 2000 y 2006).

- Factores políticos y legales (endurecimiento de gravámenes en China y legislación europea antidumping).

- Fortaleza de la moneda china (entre 2004 y 2010 el yuan se apreció casi un $13 \%$ frente al euro).

- Aumentos en los costes de logística, reflejados por ejemplo en la evolución del Baltic Dry Index que supera los 10.000 puntos en 2007 partiendo de valores en torno a 3.000 en 2003 con un máximo de 6.000 en 2005 (derivados del crecimiento del precio del petróleo y del crecimiento económico mundial). 
- Crisis económica (debilitamiento de la demanda y endurecimiento de la competencia).

La caída de la demanda en España y Europa, implica, en primer lugar, pedidos de cantidades más pequeñas, y en segundo, con plazos más cortos, debido a la debilidad financiera y la falta de liquidez de los clientes minoristas. Estos cambios son difíciles de abordar con toda la fabricación en China. Por un lado, en este país sólo es posible subcontratar grandes cantidades y con mucha antelación; por otro, las características de los sistemas logísticos encarecerían notablemente, cuando no impiden, el transporte de lotes pequeños. A todo ello hay que añadir que, en el nuevo contexto económico, las condiciones de negociación de costes de fabricación se han endurecido en China, mientras que en España son en la actualidad mucho más flexibles.

Todo ello ha conducido a las empresas a relocalizar hacia España y a algún otro país cercano toda o parte de la producción previamente deslocalizada desde el año 2010. De esta forma es factible servir pedidos más pequeños, atender reposiciones, y acortar los plazos de entrega de la mercancía. Además, ha incidido la reducción en el diferencial de los costes de fabricación entre España y Asia y el ahorro en los costes de transacción derivados de los traslados de personal propio al país extranjero para el control de la calidad del producto.

Aún así, la mayoría de las empresas mantienen parte de la fabricación en China. Sin embargo las razones son diferentes. Por una parte se encuentra una estrategia de mercado, es decir para crecer en aquel país e introducirse en nuevos mercados en expansión. Por otra, mantener allí sus estructuras productivas y comerciales introduce un elemento adicional de flexibilidad en las empresas pues, ante cambios de la situación económica, podrán reaccionar con más agilidad aumentando su producción en aquel país.

A continuación se detallan los casos de cuatro empresas paradigmáticas de los cambios que se han producido en el sector del calzado para pormenorizar los rasgos ya descritos así como mostrar la casuística que se ha ido produciendo. En primer lugar, se presenta la Tabla 1 donde se recoge la evolución de sus ingresos de explotación, de sus rentabilidades y del número de empleados para el período 1996 a 2014 de las empresas y, a continuación se detalla el proceso de deslocalización y relocalización que han seguido.

\subsection{Rebeca Sanver}

Rebeca Sanver se creó en el año 1987 fabricando calzado de señora de gama alta, cuya característica principal es la elegancia y el diseño. Simultáneamente fabricaba otra línea sin marca, de menor calidad y a un precio más bajo, denominada línea blanca. Su volumen de ventas es en 2014 de 6,7 millones de euros. La diferenciación de su producto se basa principalmente en el diseño, que 
está totalmente integrado en la empresa. Sus ventajas específicas se basan en dicha diferenciación y el prestigio de marca así como la distribución.

Tabla 1

Evolución económico-financiera de las empresas analizadas

\begin{tabular}{|c|c|c|c|c|c|c|c|c|c|c|c|c|}
\hline \multirow{2}{*}{ Año } & \multicolumn{4}{|c|}{ Ingresos de explotación (miles de euros) } & \multicolumn{4}{|c|}{ Rentabilidad Económica (\%) } & \multicolumn{4}{|c|}{ Número de empleados } \\
\hline & $\begin{array}{c}\text { Rebeca, } \\
\text { Sanver, } \\
\text { S.L. }\end{array}$ & \multicolumn{2}{|c|}{ Mustang,Gioseppo, } & $\begin{array}{c}\text { Hi-Tec } \\
\text { Sports } \\
\text { España, SA }\end{array}$ & \multicolumn{4}{|c|}{$\begin{array}{rr}\text { Rebeca, } & \text { Hi-Tec } \\
& \text { Sports }\end{array}$} & $\begin{array}{c}\text { Rebeca, } \\
\text { Sanver, } \\
\text { S.L. }\end{array}$ & \multicolumn{3}{|c|}{$\begin{array}{r}\text { Hi-Tec } \\
\text { Mustang,Gioseppo, Sports }\end{array}$} \\
\hline 1996 & 7.593 & 1.466 & 2.697 & 7.019 & 5,00 & 4,16 & 2,04 & 12,45 & 11 & n.d. & 4 & 9 \\
\hline 1997 & 9.490 & 2.437 & 2.959 & 5.683 & 4,69 & 3,96 & 4,55 & 9,66 & 13 & n.d. & 4 & 10 \\
\hline 1999 & 10.696 & 3.615 & 5.183 & 4.120 & 9,50 & $-0,77$ & 17,11 & 3,75 & 14 & n.d. & 9 & n.d. \\
\hline 2000 & 13.659 & 6.783 & 6.213 & 4.120 & 3,04 & 3,37 & 9,18 & 8,26 & 18 & n.d. & n.d. & 10 \\
\hline 2001 & 17.485 & 8.550 & 7.749 & 7.319 & 4,89 & 5,82 & 30,24 & 3,06 & 22 & 11 & n.d. & 11 \\
\hline 2002 & 21.353 & 12.316 & 10.136 & 8.384 & 5,51 & 5,57 & 32,32 & 3,50 & 22 & n.d. & 15 & n.d. \\
\hline 2003 & 21.042 & 12.352 & 13.257 & 9.822 & 9,16 & 6,26 & 33,76 & 10,38 & 23 & 20 & 20 & 15 \\
\hline 2007 & 13.488 & 36.963 & 26.491 & 14.493 & 0,46 & 46,09 & 41,66 & 15,3 & 28 & 71 & 39 & 20 \\
\hline 2008 & 12.510 & 30.591 & 25.527 & 12.777 & 1,69 & 15,45 & 22,12 & 14,82 & 34 & 76 & 56 & 22 \\
\hline 2009 & 9.654 & 34.001 & 25.359 & 12.938 & 1,11 & 41,38 & 25,59 & 13,13 & 23 & 84 & 55 & 22 \\
\hline 2010 & 7.252 & 37.804 & 30.795 & 16.270 & 0,62 & 4,83 & 14,63 & 9,41 & 21 & 91 & 63 & 24 \\
\hline 2011 & 7.251 & 58.844 & 27.830 & 13.795 & 0,72 & $-2,03$ & 8,63 & 6,19 & 20 & 124 & 71 & 21 \\
\hline 2012 & 8.457 & 56.054 & 23.895 & 12.858 & 0,77 & 0,81 & 11,14 & 3,78 & 20 & 116 & 74 & 20 \\
\hline 2013 & 7.391 & 61.380 & 27.317 & 9.217 & 0,66 & 12,63 & 17,64 & 1,58 & 22 & 114 & 87 & 18 \\
\hline 2014 & 6.671 & 66.957 & 33.527 & 11.727 & 0,39 & 26,13 & 28,40 & 6,51 & 24 & 102 & 104 & 18 \\
\hline
\end{tabular}

Fuente: Sabi, a partir de los datos depositados en el Registro Mercantil.

Desde sus orígenes, Rebeca Sanver, subcontrata casi el total de la fabricación (más del 90 por ciento) en lugar de tener fábricas propias. Inicialmente la subcontratación se realizaba a empresas que elaboraban el producto en España y desde 2006 en India y China.

En 2006, ante la competencia de calzado de origen asiático que le impide seguir compitiendo en precio, abandona la línea blanca, sigue fabricando la marca Rebeca Sanver en España y crea una nueva, To Be, de gama inferior, para fabricarla totalmente en India y posteriormente en China, logrando unos costes menores que el de su primera marca. La subcontratación de la fabricación de To Be en China la realiza con la empresa Red Dragonfly, con quien tenía un contrato previo de distribución de la marca Rebeca Sanver. De esta forma persigue crecer en el mercado chino y reforzar en el mismo la posición de su primera marca y en el exterior reforzar la posición competitiva de su segunda marca por los costes más competitivos derivados de la producción en China.

Los resultados esperados de la deslocalización eran el crecimiento de las ventas y los ingresos, lo cual permitiría incrementar la inversión en innovación, fundamentalmente en diseño, marketing y distribución, con aumentos en la 
contratación de personal cualificado en estos departamentos en España. Aunque parte de su producción se realizaba en China, la distribución comercial se realiza desde España a través de clientes minoristas, aunque mantiene contratos con diversas tiendas que venden en exclusiva su marca. Sin embargo, la empresa no consiguió los resultados esperados, ya que ni se produjeron aumentos en los ingresos ni en la rentabilidad ni en el número de empleados en los años posteriores a 2006, tal y como puede observarse en la Tabla 1 . Todo ello, unido a la caída de las ventas a partir de 2008 y al endurecimiento de las condiciones del mercado, le obligó a reconsiderar la estrategia de fabricación en China. En el nuevo contexto económico, las tiendas minoristas (los clientes de las empresas del sector calzado) demandan pequeñas cantidades de todas las colecciones y en función de cómo evolucionen las ventas, solicitan cantidades adicionales de los modelos de mayor éxito que necesitan ser servidas en plazos cortos de tiempo. La fabricación en China no parece compatible con este funcionamiento del mercado. Tanto la subcontratación en China como las necesidades del transporte requieren lotes mínimos muy superiores a los que la empresa suele atender en esa coyuntura. Además, la estructura logística hace difícil atender reposiciones en plazos cortos, aunque fuera de grandes cantidades. Por el contrario, la empresa percibe que la fabricación en España sí que le permite atender la producción de lotes más pequeños y con plazos cortos para adaptarse a la nueva situación del mercado.

Por ello, en 2011 decide relocalizar toda la fabricación en España, manteniendo el contrato de licencia de distribución en el mercado chino con la empresa local para mantener abierto un canal de ventas, sin excluir en el futuro nuevos acuerdos que pudieran suponer la fabricación en China.

Su estrategia de relocalización ha consistido, por tanto, en traer toda la fabricación a España, conservando los lazos comerciales creados en China, para mantener su presencia y seguir creciendo en aquel mercado. Pese a ello, en los años siguientes a la decisión de relocalización, de 2012 a 2014 la empresa no ha conseguido frenar la caída en sus ingresos ni en su rentabilidad.

\subsection{Mustang}

La empresa se creó en el año 1961 y adoptó la marca Mustang en 1967, produciendo calzado de señora (85 por ciento) y de caballero (15 por ciento) de gama media-alta. Su volumen de ventas alcanzó en 2014 un total de 67 millones de euros. Las ventajas de la empresa se basan en la diversificación de líneas que ofrece para cubrir distintos segmentos del mercado, todas ellas con componentes de calidad, diseño y moda. Fabricaba inicialmente toda la producción en España y desde el año 2001 subcontrata varias líneas de calzado en China.

En 2001 introdujo la marca María Mare, de gama algo menor que Mustang, subcontratando toda su fabricación en China. Con ello busca un doble objetivo: 
producir a gran escala y acceder al mercado de aquel país y de otros países distantes. Se trata, por tanto, del establecimiento de un sistema doble de producción, en España y en China.

La distribución de su producción la realiza tanto desde España como desde China, dependiendo la proporción que distribuye desde cada país de la evolución de la demanda de cada temporada de cada una de las líneas que fabrica en cada lugar. Utiliza comercios minoristas, aunque desde 2004 empezó a dar sus primeros pasos en la distribución directa, estableciendo su propia red de tiendas para el producto "outlet", con el objetivo de sentar las bases ante la posible necesidad futura de crear tiendas propias para todo el calzado que fabrica.

En el año 2005 introduce una nueva marca, Sixtyseven, la de mayor exclusividad y calidad, de gama alta. Esta línea la fábrica totalmente en España e internamente en la empresa. Desde entonces, la marca Mustang la subcontrata a empresas externas localizadas en España y en China.

La deslocalización de una de sus líneas de producto y el consecuente crecimiento de las ventas, por las ganancias de competitividad conseguidas, le ha generado importantes incrementos en sus ingresos y en su rentabilidad. Del año 2001, cuando realizó la deslocalización, al año 2002 sus ingresos se incrementaron en un 44 por ciento, pasando de 8,5 millones de euros a 12,3 y desde 2005, cuando crea la nueva marca Sixtyseven y trae la fabricación de la marca Mustang a España, hasta el año 2014 sus ingresos aumentan sobre un 140 por ciento, pasando de 27,9 millones de euros en 2005 a casi 67 euros en 2014 (Tabla 1). Asimismo su rentabilidad económica se incrementa notablemente pasando de un 5,57 por ciento en 2001 al 26,13 por ciento en 2014. Todo ello le ha permitido potenciar en España todos sus departamentos, desde la fabricación hasta el diseño, marketing y distribución, todos ellos integrados en la empresa, con incrementos continuados en el número de trabajadores, pasando de tener 11 trabajadores contratados en el año 2001 a 102 en el año 2014.

En la actualidad tiene distribuida la localización de la fabricación de sus líneas de producto entre España y China, en función a las características del producto y del destino de sus exportaciones. En los últimos años ha mantenido en Asia la fabricación de las producciones que se exportan a países distantes y está incrementando en España la fabricación del calzado que se dirige al mercado nacional y europeo, que es el de las marcas Mustang y Sixtyseven, por las mismas razones que en el caso de la empresa Rebeca Sanver. Como consecuencia de ello, se genera un proceso de relocalización continua de parte de la producción fabricada en años anteriores en China de la marca Mustang y de potenciación de producción española de la marca Sixtyseven, en función de la evolución de los mercados y la penetración de cada una de sus líneas.

Su estrategia ha consistido en mantener el sistema doble de producción, en España y en China, aumentando en los últimos años la fabricación en España, 
pero continuando con la estructura productiva en China, para poder adaptar cada año la proporción de la producción en cada país, en función de la evolución del mercado.

\subsection{Gioseppo}

La empresa Gioseppo se creó en el año 1990 fabricando sandalias de playa. Progresivamente ha ido introduciendo nuevas líneas y en la actualidad ofrece una amplia variedad de calzado de señora, caballero y niños, así como accesorios, situándose todos ellos en una gama media. Su volumen de ventas en 2014 es de 33,5 millones de euros.

La empresa tiene externalizada toda la fabricación del producto desde su inicio. Durante los primeros años se subcontrataba toda la producción en España. A partir del año 1993, deslocalizó parte de la fabricación, subcontratándola en China e India, pasando a suponer la producción en estos países el 60\% del total del volumen de negocio. Progresivamente este porcentaje aumentó hasta fabricar en el extranjero el total de la producción.

Al principio, la estrategia competitiva de la empresa se centró fundamentalmente en el precio. Paulatinamente, la empresa ha invertido gran cantidad de recursos para alcanzar prestigio de marca y actualmente la competitividad de la empresa se basa en una combinación de ambos elementos. La empresa, tras la decisión de deslocalización de la fabricación en el extranjero, centró todo su esfuerzo inversor en conseguir prestigio de marca concentrando su inversión en la creación de departamentos de diseño, marketing y publicidad, exportación, control de calidad, ventas y distribución, constituyendo esta última el pilar fundamental de la empresa.

En la actualidad puede señalarse que su ventaja competitiva tiene una doble fuente: por una parte, en una imagen de marca consolidada, que se mantiene ofertando una colección muy completa, lanzando continuamente nuevas líneas y modelos, con un gran componente de diseño y colorido; por otra, en la gestión propia de las funciones de distribución y logística.

Respecto a este segundo aspecto hay que señalar que los canales de distribución de la empresa hasta hace pocos años eran las ventas a clientes minoristas en el mercado nacional y, a través de agentes, las exportaciones indirectas a otros países. Desde 2010, Gioseppo ha ampliado los canales de venta que actualmente se componen de tres vías: Se mantiene el canal tradicional de ventas a tiendas minoristas; la empresa ha abierto tiendas propias en España y Portugal, y, por último, ha creado e impulsado una línea de distribución on-line para acceder directamente al cliente final.

El resultado ha sido un incremento continuado de sus ingresos y de su rentabilidad, sólo con un descenso de esta última en el año 2011 pero con 
crecimiento en los siguientes años. Los ingresos pasan de 2,7 millones de euros en 1996 a 33,5 en 2014 y la rentabilidad de 2,04 por ciento en el primer año a 28,4 por ciento en 2014.

En 2010 la empresa inició una estrategia de relocalización de una parte de la fabricación que tenía subcontratada en China, tanto a España como a Portugal a partir de 2011. Las razones son las mismas que en los casos descritos previamente. Este cambio supuso un crecimiento notable en su tamaño que pasa de 63 trabajadores en 2010 a 104 en 2014 (Tabla 1). Para explotar los beneficios que se derivan del crecimiento del mercado chino, la empresa se plantea como objetivo a corto plazo de distribuir una parte de la fabricación que permanece en China directamente desde allí a aquel mercado, contratando personal específico para realizar dicha actividad.

\subsection{Hi-Tec Sports España, S.A.}

Hi-Tec Sports es una empresa multinacional de calzado creada en el año 1974, con sede central en Holanda y con distintas filiales en países europeos. Uno de ellos es España, donde está instalada Hi-Tec Sports España, S.A. desde el año 1992. Tiene dos divisiones, una de ellas es Hi-Tec, de calzado deportivo de montaña, y la otra Magnum, de calzado militar. Su volumen total de ventas en España es 11,7 millones de euros en el año 2014.

La empresa española (así como el resto de empresas del grupo), ha subcontratado desde su inicio todo el proceso de fabricación del calzado, y ha centrado su actividad interna en el desarrollo del producto, el diseño, la distribución y la gestión. Su estrategia ha sido siempre localizar la fabricación en aquellos países donde se dieran las condiciones más favorables para obtener las calidades perseguidas en el tipo de calzado que desarrolla. La filial española ha localizado hasta 2010 toda la fabricación en China, Indonesia y Vietnam, si bien desde entonces ha empezado a fabricar en España una de las líneas de calzado. Sus mercados son, además de España, las exportaciones que realiza a través de redes propias de ventas en Francia y Portugal.

La competitividad de su calzado se deriva fundamentalmente de los factores de diferenciación basados en el diseño, calidad y marca, además del precio, si bien éste último tiene un carácter secundario en este segmento del mercado. La empresa basa su ventaja comparativa en el prestigio de su marca, respaldada por la calidad, y el trato a los clientes. Hay que señalar que, a diferencia del calzado de vestir, en el segmento de calzado deportivo el reconocimiento de la marca es el elemento fundamental para competir en el mercado.

La distribución del calzado deportivo se realiza de forma diferente a como lo hace en el calzado de vestir. En el zapato de vestir las tiendas demandan cantidades pequeñas de cada modelo al principio de la temporada realizándose pedidos adicionales en función de la evolución de la campaña. Por el contrario, 
en el calzado deportivo se realizan grandes envíos al principio de cada temporada y no hay prácticamente reposiciones.

La naturaleza de la distribución del calzado deportivo hace que una localización geográfica lejana de la fabricación no constituya una limitación cuando las circunstancias del mercado son favorables. Sin embargo, la coyuntura económica caracterizada por el debilitamiento de la demanda y la falta de liquidez de los clientes minoristas convierte a los plazos de entrega en un factor de competitividad importante. La fabricación en China requiere contratar el volumen de producción con un plazo de tiempo muy largo, previo al comienzo de la temporada. Esto implica que los clientes minoristas han de hacer los pedidos con varios meses de antelación y recibir la mercancía y pagarla, también con un plazo de antelación relativamente largo al comienzo de la temporada y de las ventas. Por su parte, la fabricación en España conlleva la ventaja que supone unos plazos de entrega más cortos. De esta forma, los clientes (vendedores minoristas) pueden realizar los pedidos, recibir la mercancía y financiarla en un plazo de tiempo más corto previo al inicio de la temporada. La empresa percibe que la fabricación en España supone una mejora en el servicio a sus clientes, que pasa a constituir uno de los pilares de la competitividad de la empresa: la mejora en la distribución.

Por esta razón, desde 2010 la empresa española ha decidido relocalizar en España una línea de calzado que se fabricaba en China. Se trata de una línea sencilla, que no requiere elevada cualificación de la mano de obra, por lo que se pueden obtener en el país nacional los mismos niveles de calidad que en el extranjero. Hay que señalar que en el caso del calzado deportivo y, al contrario que en el de vestir, la cualificación de la mano de obra es superior en los países asiáticos que en España. Esta relocalización no supuso un aumento en el número de trabajadores de la empresa (Tabla 1) puesto que la fabricación sigue siendo subcontratada, pero a empresas ubicadas en España.

La evolución de sus ingresos es creciente, con alguna caída puntual en los años 2008, 2009, 2011 y 2013, como consecuencia de la caída en las ventas derivadas de la crisis económica.

En la Tabla 2 se resumen los cuatro casos presentados, en la que se puede ver la importancia que en todos ellos tuvo la estrategia de deslocalización en la década de los noventa y primeros años del siglo XXI que a partir de 2010 fue deshecha para traer la producción de nuevo a España. Como se sintetiza en la tabla y queda reflejado en el análisis de cada caso, la relocalización no parece deberse a la consideración de un error de la deslocalización que previamente se había producido, sino a la necesidades que el nuevo entorno económico plantea y para el que la proximidad al cliente final (debido a las características específicas de la distribución del producto) es un factor importante de competitividad. 
Tabla 2

Estrategias de deslocalización y relocalización de las empresas

\begin{tabular}{|c|c|c|c|c|c|}
\hline Empresa & Tipo de calzado y Marcas & $\begin{array}{c}\text { Deslocalización } \\
(1993-2006)\end{array}$ & $\begin{array}{l}\text { Producción en } \\
\text { España tras la } \\
\text { deslocalización } \\
(2000-2006)\end{array}$ & $\begin{array}{c}\text { Relocalización } \\
\text { 2010- }\end{array}$ & $\begin{array}{c}\text { Estrategia } \\
\text { 2011-12 }\end{array}$ \\
\hline $\begin{array}{l}\text { Rebeca } \\
\text { Sanver }\end{array}$ & $\begin{array}{l}\text { Rebeca Sanver: gama alta } \\
\text { Línea blanca } \\
\text { To Be: gama media }\end{array}$ & $\begin{array}{l}2006 \\
\text { China e India } \\
\text { To Be }\end{array}$ & Rebeca Sanver & $\begin{array}{l}2011 \\
\text { España } \\
\text { Toda la producción }\end{array}$ & $\begin{array}{l}\text { Relocalizar en España todo } \\
\text { lo deslocalizado } \\
\text { previamente. } \\
\text { Mantenimiento de la red } \\
\text { comercial licenciada. }\end{array}$ \\
\hline Mustang & $\begin{array}{l}\text { Mustang: gama media-alta } \\
\text { María Mare: gama media } \\
\text { Sixtyseven: gama alta }\end{array}$ & $\begin{array}{l}2001 \\
\text { China } \\
\text { María Mare } \\
\text { Una parte de } \\
\text { Mustang }\end{array}$ & $\begin{array}{l}\text { Una parte de Mustang } \\
\text { Nueva marca } \\
\text { Sixtyseven }\end{array}$ & $\begin{array}{l}2011 \\
\text { Toda la producción de } \\
\text { Mustang }\end{array}$ & $\begin{array}{l}\text { Sistema mixto de } \\
\text { fabricación, en China y } \\
\text { España, variando la } \\
\text { distribución de las } \\
\text { cantidades en cada país en } \\
\text { función de la demanda. } \\
\text { Sixtyseven todo en España. }\end{array}$ \\
\hline Gioseppo & Gioseppo: gama media & $\begin{array}{l}1993 \\
\text { China } \\
\text { Una parte de la } \\
\text { producción }(60 \%) \\
2010 \text { (100\% de la } \\
\text { producción) }\end{array}$ & Nula & $\begin{array}{l}2009- \\
\text { Parte de la producción } \\
\text { en España. } \\
2010- \\
\text { Parte de la producción } \\
\text { en Portugal }\end{array}$ & $\begin{array}{l}\text { Relocalización de parte de } \\
\text { la producción en España y } \\
\text { países cercanos. } \\
\text { Creación de redes de } \\
\text { distribución propias. En } \\
\text { España, China y Portugal. }\end{array}$ \\
\hline $\begin{array}{l}\text { Hi-Tec } \\
\text { Sports } \\
\text { España, } \\
\text { S.A. }\end{array}$ & $\begin{array}{l}\text { Hi-Tec: calzado deportivo de } \\
\text { montaña. Gama alta } \\
\text { Magnum: calzado militar. } \\
\text { Gama alta }\end{array}$ & $\begin{array}{l}\text { Toda la fabricación } \\
\text { subcontratada } \\
\text { desde el inicio en } \\
\text { Indonesia, China y } \\
\text { Vietnam }\end{array}$ & Nula & $\begin{array}{l}2010- \\
\text { Una línea de calzado } \\
\text { sencilla }\end{array}$ & $\begin{array}{l}\text { Localización de la } \\
\text { producción en los países } \\
\text { más favorables. } \\
\text { Relocalización de una parte } \\
\text { de la fabricación extranjera } \\
\text { a España. }\end{array}$ \\
\hline
\end{tabular}

Fuente: Elaboración propia.

\section{CONCLUSIONES}

El aumento en la competencia en los mercados ha forzado a las empresas a la explotación al máximo de la estrategia de localización de las distintas fases de sus procesos productivos. La apertura a las inversiones exteriores, la reducción de las barreras al comercio internacional, los cambios en los costes de transporte, las nuevas tecnologías que favorecen la búsqueda de suministradores y la gestión de la relación con los mismos, etc. han favorecido el desplazamiento de ciertas actividades productivas hacia países como China donde los menores costes laborales suponen una ventaja competitiva en aquellas fases del proceso productivo intensivas en trabajo. Sin embargo, en los últimos años se observa que se están produciendo casos de 'relocalización' de algunas de estas actividades, volviendo a sus países de origen. En este trabajo se analiza este fenómeno de la relocalización de las empresas del sector calzado español. Se trata de una actividad que previamente fue deslocalizada desde España (así como otros países) hacia China e India con el fin de ganar competitividad gracias a los menores costes de la mano de obra que es el principal factor productivo.

En este trabajo se recoge un análisis que se ha realizado a empresas de la industria zapatera española. Se han seleccionado las empresas más importantes, por volumen de negocio, de la provincia de Alicante, donde se concentra más 
del sesenta por ciento de la fabricación de calzado nacional. El análisis utiliza información cualitativa recogida en una serie de entrevistas a directivos de cuatro empresas que resumen la casuística de todas las empresas analizadas.

La conclusión general es que las relocalizaciones se deben a un conjunto de factores externos que se han generado recientemente como consecuencia de la crisis económica, y que no estaban presentes cuando se adoptaron las decisiones de deslocalización. Estos incluyen por un lado, el menor atractivo que presenta la producción en Asia al haberse reducido el diferencial de costes laborales como consecuencia de la crisis económica en España y los incrementos de costes salariales en China. Por otro lado, se encuentran factores específicos de la actividad logística y de distribución. Dicha actividad tiene un gran peso en los costes totales ya que, al producir en China, las empresas deben transportar toda la producción a España desde donde se distribuye por la propia empresa. Más allá del coste estricto de transporte, las empresas destacan que tener localizada la producción cerca del mercado les permite reducir los plazos de entrega del producto al cliente minorista y atender pedidos de menor tamaño. Ambos factores suponen una desventaja (tanto por los costes logísticos como por la naturaleza de los contratos de outsourcing) en el caso de localizar la producción en China. Estos elementos constituyen un factor clave de la estrategia competitiva en relación con la distribución que se ha convertido en uno de los factores clave para las empresas de la industria del calzado, máxime en un entorno en el que las mejoras en el servicio a los clientes minoristas constituye uno de los factores determinantes para conservar su fidelidad.

La unión de ambos elementos ha conducido a diferentes empresas del sector calzado a la reconsideración de la estrategia de outsourcing internacional, llevándolas a volver a fabricar una parte o toda la producción en España. Sin embargo, como ya se ha señalado esta relocalización no es consecuencia de una corrección de errores en la decisión inicial de fabricación en el extranjero, sino a los cambios producidos posteriormente en el entorno, en una magnitud difícilmente previsible. De hecho, las deslocalizaciones generaron resultados positivos para las empresas, ya que les proporcionó importantes ventajas competitivas vía precios lo que posibilitó su expansión, nacional e internacional. Asimismo, al externalizar la fabricación en otros países y explotar así las ventajas comparativas de éstos en términos de costes laborales, concentraron sus inversiones en lo que pasaría a constituir su "núcleo de negocio" basado en la explotación de la marca, diseño, calidad, marketing y distribución, potenciando así dichas funciones, totalmente internalizadas aumentando el personal contratado con elevada cualificación. Obviamente, ello tuvo impacto negativo sobre el empleo menos cualificado dedicado a la fabricación. Sin embargo, los procesos de relocalización actuales podrían compensar parcialmente dichas pérdidas. 
Los resultados obtenidos en este estudio coinciden parcialmente con los que se deducen de la evidencia empírica previa referida a otras industrias y otros países. En el conjunto de trabajos publicados reseñados en Stentoff (2016) se señalan todos los factores encontrados que han motivado las relocalizaciones en cada caso. Las causas aquí descritas para el sector del calzado también se presentan en otros estudios referidos a otras industrias, tales como el automóvil o las bicicletas donde la importancia de la flexibilidad en la distribución se revela como factores importantes. Por su parte, Hammer y Plugor (2016) apuntan también a la mayor necesidad de lotes de menor tamaño con una mayor variabilidad como factores explicativos en los casos detectados de relocalización de empresas del sector textil del Reino Unido. Sin embargo, los estudios de una perspectiva sectorial más amplia (como los referenciados en Kinkel, 2014) muestran que las causas relevantes de la relocalización son diferentes, destacando factores como como la imposibilidad de alcanzar en el país extranjero los mismos niveles de calidad y de productividad que en el país nacional o los elevados costes de transacción derivados del excesivo esfuerzo de coordinación que implica la producción en el país extranjero, lo que apunta a que la relocalización responde a una corrección de una decisión que no valoró adecuadamente todos los elementos relevantes en dicha estrategia.

Finalmente, hay que señalar que estas conclusiones abren nuevas líneas de investigación. En primer lugar, es necesario conocer en qué medida los factores que explican la relocalización de la actividad productiva en la industria del calzado pueden promover la relocalización en otras industrias con rasgos similares. En segundo lugar, la comparación internacional con otros países que en el pasado hayan deslocalizado la producción de calzado a países asiáticos permitirá dar robustez a los resultados. Finalmente, se abre un campo de trabajo para determinar si estos cambios permiten consolidar la producción del sector localizada en España de forma competitiva o tan sólo en la coyuntura actual de crisis de demanda.

\section{REFERENCIAS BIBLIOGRÁFICAS}

ALAN, G.C. (2004). "Offshoring in the Service Sector: Economic Impact and Policy Issues". Economic Review - Federal Reserve Bank of Kansas City, 89(3), pp. 5-37.

AMIGHINI, A. y RABELLOTTI, R. (2010). "How Do Italian Footwear Industrial Districts Face Globalization". European Planning Studies, 14(4), pp. 485-502.

ARAUZO-CAROD, J., LIVIANO-SOLIS, D. y MANJON-ANTOLÍN, M. (2010). "Empirical studies in industrial location. An assessment of their methods and results". Journal of Regional Science, 50(3), pp. 685-711. 
ARNOLD, U. (2000). "New dimensions of outsourcing cost economics and the core competence concept". European Journal of Purchasing and Supply Management, 6, pp. 23-39.

BARNEY, J.B. (1991). "Firm resources and sustained competitive advantage". Journal of Management, 17 (1), pp. 99-120.

BALDWIN, R. y VENABLES, A.J. (2013). "Spiders and snakes: Offshoring and agglomeration in the global economy". Journal of International Economics, 90, pp. 245254.

BOSTON CONSULTING GROUP (2011). "Made in America, Again. Towards a developing country firm perspective on outsourcing". Ed. Boston Consulting Group.

CHRISTOPHERSON, S., MARTIN, R., SUNLEY, P. and TYLER, P. (2014). "Reindustrialising regions: rebuilding the manufacturing economy?". Cambridge Journal of Regions, Economy and Society, 7, pp. 351-358.

CUTRINI, E. (2011). "Moving Eastwards While Remaining Embedded: The Case of the Marche Footwear District, Italy". European Planning Studies, 19(6), pp. 991-1019.

DELGADO, M.; PORTER, M. y STERN, S. (2010). "Clusters and entrepreneurship". Journal of Economic Geography, 10, pp. 495-518.

DUNNING, J.H. (1980). "Towards an eclectic theory of international production: some empirical tests". Journal of International Business Studies, 22(2), pp. 9-31.

EISENHARDT, K.M. (1989). "Building theories from case study research". Academy of Management, 14(4), pp. 532-550.

ELLRAM, L.M., TATE, W. y PETTERSEN, K.J. (2013). "Offshoring and reshoring: An update on the Manufacture Location Decision". Journal of Supply Chain Management, 46(2), pp. 14-22.

FRATOCCHI, L. ANCARANI, A., BARBIERI, P., DI MAURO, C., NASSIMBENI, G. SARTOR, M., VIGNOLI, M. y ZANONI, A. (2016). "Motivations of manufacturing reshoring: an interpretative framework". International Journal of Physical Distribution \& Logistics Management, 46(2) pp. 98-127.

FREYGTAG, P.V.; CLARKE, A.H. y EVALD M.R. (2012). "Reconsidering outsourcing solutions". European Management Journal, 30, pp. 99-110.

FUSTER, B.; MARTÍNEZ, C. y PARDO, G. (2008). "Las estrategias de competitividad de la industria de calzado ante la globalización". Revista de Estudios Regionales, 86, pp. 7196.

GADDE, L. E. y JONSSON, P. (2009). "Outsourcing and supply network performanceConsequences of sourcing and producing in low-cost countries". IMP Conference, http://impgroup.org/

GAGLIARDI, L. IAMMARINO, S. y RODRÍGUEZ-POSE, A. (2015). Offshoring and the geography of Jobs in Great Britain. CEPR Discussión Paper 10855.

GANDOY, R. (2017). "La dependencia importadora de la economía española". Estudios de Economía Aplicada, vol. 35(1), pp. 111-132.

GYLLING M, HEIKKILÄ J, JUSSILA K, y SAARINEN M (2015). "Making decision on offshore outsourcing and backshoring: a case study in the bicycle industry". International Journal of Production Economics, 162, pp. 92-100.

HAMMER, N. y PLUGOR, R. (2016). "Near-sourcing UK apparel: value chain restructuring, productivity and the informal economy". Industrial Relations Journal 47(5-6), pp. 402416. 
HANSEN, M.W., SCHAUMBURG-MÜLLER, H. y POTTENGER, E. (2008). "Towards a developing country firm perspective on outsourcing". Strategic Outsourcing: An International Journal, 1(3) pp. 210-229.

KINKEL S. (2012). "Trends in production relocation and backshoring activities: changing patterns in the course of the global economic crisis". International Journal of Operations \& Production Management, 32(6), pp. 696-720.

KINKEL, S. (2014). "Future and impact of backshoring - Some conclusions of 15 years of research on German practices". Journal of Purchasing \& Supply Management, 20, pp. 63-65.

LABRIANIDIS, L. y KALANTARIDIS, C. (2004). "The delocalization of production in labour intensive industries: Instances of triangular manufacturing between Germany, Greece and FYROM". European Planning Studies, 72(8), pp. 1157-1173.

LOWDER, S. (1999). "Globalization of the footwear industry: A simple case of labour?" Tijdschrift voor economische en sociale geografie, 90(1), pp. 47-60.

MITZA, T. y KREUZER, F. (2015). Is there a curse of relocation? Analysing the causal link between offshoring and the innovation performance of (small) firms. Bulletin of Economic Research (in press) DOI: 10.1111/boer.12064.

MOSER, H. (2013). "Manufacturing: reshoring tools to retain companies and attract foreign direct investment". Economic Development Journal, 12(1), pp. 5-11.

MYRO, R. y LABRADOR, L. (2008). "Deslocalización: concepto, formas y efectos". Principios: Estudios de Economía Política, 10, pp. 97-107.

MYRO, R.; FERNÁNDEZ-OTHEO, C.M.; LABRADOR, L.; BAIDES, A.B.; ÁLVAREZ, E. y VEGA, J. (2008). Globalización y deslocalización. Importancia y efectos para la industria española. Madrid: Ministerio de Industria, Turismo y Comercio.

SÁNCHEZ-ROBLES, B. (2006). "Intermediate goods imports, policy and growth. A simple theoretical model". Estudios de Economía Aplicada, vol. 24(1), pp. 105-126.

STENTOF, J.; OLHAGER, J.; HEIKKILA, J. y THOMS, L. (2016). "Manufacturing backshoring: a systematic literature review". Operations Management Research, 9, pp. 53-61.

TIMMER, M.P.; LOS, B.; STEHRER, R. y DE VRIES, G.J. (2016). An Anatomy of the Global Trade Slowdown based on the WIOD 2016 Release. GDC Research Memorandum, $\mathrm{n}^{\circ} 162$.

VELTRI, N.; CAROL, F.; SAUNDERS, S. y KAVAN, C. B. (2008). "Information systems backsourcing: Correcting problems and responding to opportunities". California Management Review, 51(1), pp. 50-76.

WILLIAMSON, O. E. (1975). Markets and hierarchies. New York: Free Press.

WRIGTH, G.C. (2014). "Revisiting the employment impact of offshoring". European Economic Review, 66, pp. 63-83. 
